\title{
$O$ atendimento odontológico em pacientes submetidos a radioterapia de cabeça e pescoço: revisão de literatura
}

\author{
Dental care for patients undergoing head and neck radiotherapy: literature review \\ El atendimiento odontológico en pacientes submetidos a radioterapia de la cabeza y del \\ cuello: revisión de literatura
}

André da Silva Fernandes ${ }^{1 *}$, Brenna Cristiny Leite de Oliveira ${ }^{1}$, Lays Melyssa Costa da Silva ${ }^{1}$, Diogo Henrique Ohse², Yuri da Silva Pimenta².

\begin{abstract}
RESUMO
Objetivo: Mostrar através de uma revisão narrativa sobre a importância da atuação do cirurgião dentista no manejo de pacientes oncológicos para o melhor diagnóstico e adequação da cavidade oral, anterior ao início do tratamento, durante e posteriormente ao tratamento da radioterapia. Revisão Bibliográfica: A radioterapia é um tratamento não-seletivo e pode afetar tanto células neoplásicas (com alterações teciduais) como células saudáveis, o que a torna tóxica ao organismo. As alterações adversas causadas pela radioterapia em região de cabeça e pescoço observadas em pacientes que são submetidos ao tratamento antineoplásico são a mucosite oral, disgeusia, xerostomia, dermatite aguda, cárie por radiação e osteorradionecrose. Estas doenças levam à perda significativa da qualidade de vida do paciente. Logo, esta revisão pretende analisar e compreender a causa, prevenção e o tratamento das sequelas decorrentes da radioterapia. Considerações Finais: É fundamental o acompanhamento odontológico que anteceda o tratamento radioterápico como forma de prevenir e diminuir a incidência de complicações bucais e infecções oportunistas.
\end{abstract}

Palavras-chave: Radioterapia, Tratamento odontológico, Osteorradionecrose, Terapia.

\begin{abstract}
Objective: Show through a narrative review on the importance of the dentist's role in the management of cancer patients for the best diagnosis and adequacy of the oral cavity before the beginning of treatment, during and after the radiotherapy treatment. Bibliographic Review: Radiotherapy is a non-selective treatment and can affect both neoplastic cells (that has tissue alteration) and healthy cells, which makes it toxic to the body. The adverse changes caused by radiotherapy in the head and neck region observed in patients who are undergoing antineoplastic treatment are oral mucositis, dysgeusia, xerostomia, acute dermatitis, radiation induced caries and osteoradionecrosis. These diseases lead to a significant loss of the patient's quality of life. Therefore, this review aims to analyze and understand the cause, prevention and treatment of sequelae that are resulting from radiotherapy. Final Considerations: Dental follow-up prior to radiotherapy is essential to prevent and reduce the incidence of oral complications and opportunistic infections.
\end{abstract}

Keywords: Radiotherapy, Dental treatment, Osteoradionecrosis, Therapy.

\section{RESUMEN}

Objetivo: Muestra a través de una revisión narrativa sobre la importancia de la actuación del cirujano dentista en el manejo de pacientes oncologos para el mejor diagnóstico y adecuación de la cavidad oral, anterior al inicio del tratamiento de la radioterapia. Revisión Bibliográfica: La Radioterapia es un tratamiento no selectivo y puede afectar tanto células neoplásicas (con alteraciones en los tejidos) como en las células saludables, que lo hace tóxica al organismo. Las alteraciones adversas ocasionadas por la radioterapia en la región de la cabeza y en el cuello observadas en el paciente que son submetidos al tratamiento antineoplásico so de la mucositis oral, disgeusia, xerostomía, dermatitis aguda, caries por radiación, osteorradionecrosis. Estas enfermedades llevan a la pérdida significativa de la cualidad de vida del paciente. Luego, esta visión pretende analizar y comprender a la causa, prevención y el tratamiento de las secuelas resultantes de la radioterapia. Consideraciones Finales: Es fundamental el acompañamiento odontológico que precede el tratamiento radioterápico con la forma de prevenir y dimunuir la incidencia de complicaciones orales y infecciones oportunistas.

Palabras clave: Radioterapia, Tratamiento odontológico, Osteorradionecrosis, Terapia.

${ }^{1}$ Centro Universitário do Norte (UNINORTE), Manaus - AM. *E-mail: andrefernandesfpf@gmail.com

2 Universidade Federal do Amazonas (Ufam), Manaus - AM. 


\section{INTRODUÇÃO}

As neoplasias que acometem a região da cabeça e pescoço são alterações teciduais resultantes de um crescimento desordenado das células. De acordo com o Instituto Nacional do Câncer (INCA) elas podem ser classificadas em: Neoplasias Benignas (NB) e Neoplasias Malignas (NM). As NB são células de crescimento lento, com limites definidos, não apresentam potencial de metástases e não costumam recidivar; e as neoplasias malignas (NM), conhecidas popularmente como tumor ou câncer, possuem um crescimento celular anormal, capacidade de invaginação tecidual, metástases e grandes chances de recidiva (NEVILLE B, et al., 2016).

O câncer de cabeça e pescoço representa $6 \%$ de todos os cânceres diagnosticados mundialmente, sendo a nível global sexto mais comum, com cerca de 600.000 novos casos e 350.000 mortes por ano. Estima - se que em 2020 a incidência dessa neoplasia aumentará 30\% devido ao envelhecimento da população mundial (CARVALHO DA, et al., 2019).

As lesões podem acometer os seguintes sítios anatômicos: cavidade oral, faringe, cavidade nasal, seios paranasais, laringe, glândulas salivares. No manejo dessas patologias, podem ser empregadas formas de tratamento como a cirurgia, a quimioterapia e a radioterapia (DAVID EF, et al., 2016).

A radioterapia (RT), muitas das vezes, é utilizada para o controle e diminuição das lesões malignas da cabeça e pescoço ou no auxílio de um tratamento cirúrgico. É uma das formas de tratamento feito in loco regional, podendo resultar em alterações nos tecidos vizinhos aos irradiados (NEVILLE B, et al., 2016).

As doses de irradiação no tratamento de NM em região de cabeça e pescoço podem chegar até 7000 Gy. A escolha do protocolo de tratamento varia de acordo com a lesão e quanto mais potente o tratamento, maior o risco de complicações (NEVILLE B, et al., 2016; CARVALHO DA, et al., 2019).

As alterações causadas pelas irradiações podem ser classificadas em injúrias agudas, quando se observa logo após o tratamento de RT e injúrias tardias, quando se apresentam depois de um certo tempo após a RT. As injúrias agudas são: mucosite, disgeusia, xerostomia e descamação da pele. Já as injúrias tardias são: ulceração da mucosa, lesões vasculares, atrofia dos tecidos, perda ou mudança do paladar, fibrose, edema, necrose dos tecidos moles, perda de dentes, diminuição do fluxo salivar, osteorradionecrose e cárie de radiação (ROLIM AEH, et al., 2011).

Este trabalho tem como objetivo realizar uma revisão de literatura para trazer informações ao cirurgião dentista quanto ao manejo odontológico a pacientes que são submetidos a radioterapia de cabeça e pescoço. Logo, esta revisão pretende analisar e compreender a causa, prevenção e o tratamento das sequelas decorrentes da radioterapia.

\section{REVISÃO BIBLIOGRÁFICA}

\section{Radioterapia}

A Radioterapia é uma modalidade de tratamento principal ou adjunta que ao longo dos anos tem se mostrado bastante eficaz no tratamento do câncer com o objetivo de erradicar a lesão, dar uma melhor qualidade de vida ao paciente e aumentar a taxa de sobrevivência. A radioterapia consiste na utilização de energia eletromagnética ionizante ou corpuscular que por sua vez tem a capacidade de interagir com os tecidos no tratamento do câncer. Os elétrons produzidos pela radiação são deslocados nos tecidos e ionizam o meio provocando uma reação química que resulta em danos no ácido desoxirribonucleico (DNA) que impedem a replicação de células neoplásicas, este tratamento não é seletivo podendo afetar tanto células neoplásicas como células saudáveis o que torna tóxico ao organismo (BARBIERI T, et al., 2020).

$\mathrm{Na}$ radioterapia a unidade de medida que se usa para avaliar o nível de radiação é gray (Gy) que equivale $1 \mathrm{~Gy}=1 \mathrm{~J} / \mathrm{kg}=100$ rads. A dose de radiação em que o paciente é submetido no tratamento do câncer na região de cabeça e pescoço geralmente é de 50 e 70 Gy. Essas doses são aplicadas de forma fracionada num espaço de tempo de cinco a sete semanas fazendo o uso de uma vez ao dia e cinco dias na semana com uma dose diária na lesão em torno de 2 Gy, mesmo utilizando doses baixas de radiação como de 10 Gy já há aparecimentos de alterações como mucosite e alterações das glândulas salivares (ROLIM AEH, et al., 2011; CARVALHO DA, et al., 2019; FRANCO R, et al., 2020). 
As doses de irradiações elevadas no tratamento de uma neoplasia podem causar alterações químicas, físicas e biológicas ao nível tecidual e celular pela ação direta e erradicação da microcirculação local. Os efeitos positivos desta terapia dependerão da capacidade das células, tecidos e órgãos de se reparar, reabastecer e reoxigenar. Com isso as alterações causadas variam de acordo com a resposta biológica de cada paciente, da área irradiada e quantidade de radiação utilizada no tratamento.

Segundo Rolim AEH, et al. (2011) devem-se considerar determinadas situações modificadoras: as de consumo e as que comprometem a integridade da mucosa bucal. As primeiras dizem a respeito ao consumo de álcool e tabaco e a segunda às condições bucais do paciente, como uma prótese mal adaptada, cárie e doença periodontal preexistentes. Também se consideram os hábitos de higiene ou falta deles, estado de restauração e tratamento endodôntico realizado e conscientização e cooperação do paciente durante a radioterapia.

\section{Mucosite}

Uma das alterações adversas causadas pela radioterapia em região de cabeça e pescoço e observado em pacientes que são submetidos ao tratamento antineoplásico é a mucosite oral (MO), um importante efeito adverso observado. Esta doença se apresenta clinicamente por lesões erosivas e/ou ulcerativas, podendo causar dor leve a grave, geralmente levam à perda significativa da qualidade de vida uma vez que podem prolongar o tempo de internação hospitalar, influenciar no estado nutricional do paciente e aumentar o risco de infecções. Desta forma, o tratamento da MO é extremamente necessário com o objetivo de aliviar os sintomas, acelerar o reparo tecidual e controlar eventuais infecções de origem bucal. Atualmente, esforços estão focados na prevenção da MO (EDUARDO FP, et al., 2011; HOLMES TSV, et al., 2014; CURRA M, et al., 2018).

Um dos tratamentos recomendados para o manejo da mucosite é a laserterapia. O principal efeito desse procedimento é a diminuição do tempo de duração da inflamação aguda podendo fazer com que todo o processo de cicatrização das feridas ocorra de forma mais rápida sabendo que a luz a lazer faz com que a atividade celular seja estimulada assim como consequencia ajuda a propiciar a liberação de fatores de crescimento por macrófagos e proliferação de queratinócitos, como também prolifera a população e a degranulação de mastócitos e promovendo a angiogênese. Ademais, a aplicação diária reduz a duração e a intensidade da mucosite, diminuindo assim a dor do paciente, e por consequência, a administração de morfina (REOLON LZ, et al., 2017; LOPES LD, et al., 2019).

\section{Disgeusia}

A distorção persistente da sensação gustativa (disgeusia), sendo transitória ou permanente, pode ter influência significativa no cotidiano dos pacientes submetidos a RT e apresenta-se como perda ponderal, anorexia, desnutrição, quadros depressivos e redução significativa da qualidade de vida. Segundo Barros $O$ et al. (2015) alterações na função gustativa, quer transitórias quer permanentes, podem ter consequências importantes para o cotidiano dos indivíduos, estando relacionadas com alterações ponderais e quadros depressivos.

\section{Xerostomia}

Este tipo de patologia é definido como uma sensação subjetiva de boca seca, sendo encadeada como consequência da redução da produção de saliva chamada de hipossalivação. A xerostomia é responsável pela sensação de boca seca e ardor na boca que são as queixas frequentes dos pacientes com este tipo de alteração. Embora seja uma alteração comum e extremamente desagradável, o controle da xerostomia e o hipofluxo salivar consistem principalmente em tratamentos paliativos, incluindo a aplicação de substâncias e estimulantes, terapia a laser de baixa potência e medicamentos prescritos (BARBIERI T, et al., 2020).

O tratamento da xerostomia é essencialmente paliativo e é descrita uma relação direta entre a dose de irradiação e a extensão das modificações glandulares, que pode ser feito por meio de uso de estimulantes mecânicos/gustatórios, substitutos da saliva ou agente sistêmicos. Segundo Freitas DA, et al. (2011) pacientes com esta patologia devem ingerir de 8 a 12 copos de água por dia e alimentos ricos em ácido ascórbico, ácido málico ou ácido cítrico, ao passo que devem evitar café, tabaco, álcool e alimentos e bebidas com muito açúcar ou muito sal. 


\section{Dermatite aguda}

De acordo com Neville B, et al. (2016) a dermatite aguda por radiação é comum e varia de acordo com a intensidade do tratamento. A radiação moderada causa eritema e edema combinados com perda de pele e úlceras. Quando se torna crônica, é caracterizada por áreas brilhantes, atróficas e necróticas, com telangiectasia (vasos muito finos na superfície da pele), desaparecimento de estruturas foliculares ou úlceras. Os pacientes devem cuidar bem da pele, mantendo-a bem hidratada e usar proteção solar para evitar ainda mais danos ao tecido.

\section{Cárie por radiação}

A cárie por radiação é uma alteração que se desenvolve após a RT e é caracterizada por rápido desenvolvimento, geralmente na região cervical do dente. Desenvolve-se de forma mais acelerada que a cárie convencional, pois atinge a dentina em um mês, enquanto a cárie convencional leva um ano, em média, em pacientes não irradiados. Acreditasse que a cárie por radiação é resultado da alteração na composição química da saliva e desenvolvimento de microrganismos cariogênicos. Além disso, a hipossalivação é sempre acompanhada por uma mudança nos hábitos alimentares, os alimentos pastosos ricos em carboidratos são consumidos com mais frequência, o que favorece o desenvolvimento de cárie por radiação, no entanto, ela pode ser resultado não apenas dos efeitos indiretos da RT, mas também de efeitos diretos nos dentes (ROLIM AEH, et al., 2011; SARI J, et al., 2014).

\section{Osterradionecrose}

A osteorradionecrose (ORN) é uma complicação grave e severa advinda do tratamento da RT, podendo acontecer tanto na mandíbula quanto na maxila, sendo mais comumente encontrado na região de mandíbula. Esta patologia caracteriza-se pela exposição do osso desvitalizado em que não houve reparo tecidual e celular no mínimo entre três a seis meses no momento do diagnóstico, na ausência de doença neoplásica local. A ORN pode se apresentar de forma espontânea ou devido à doença periodontal, doença apical, trauma induzido por próteses, cirurgias ou extrações dentárias. A remodelação óssea é um processo fisiológico que equilibra a deposição (atividade osteoblástica) e a reabsorção (atividade osteoclástica) do tecido. Quando há um desequilíbrio entre essas atividades, o processo patológico ORN é estabelecido. Os sinais e sintomas clínicos incluem necrose óssea, dor, disgeusia, fístula oroantral, odor fétido, trismo, fístula extraoral (DIAS LL, et al., 2018; RIBEIRO GH, et al., 2018).

Existem várias formas de tratamento da ORN para restaurar a vascularização do osso, permitindo que o processo de cicatrização da lesão ocorra e mantenha a homeostase tecidual. Os critérios para o sucesso são uma condição assintomática estável, função normal, contorno ósseo normal, manutenção da mucosa oral subjacente. A escolha do tratamento é determinada pela gravidade da ORN. Existem várias formas de tratamento da ORN uma das formas de tratamento é baseado no tratamento cirúrgico onde consiste na ressecção do osso necrótico dos tecidos moles e reconstrução primaria, depois do tratamento cirúrgico opções viáveis de reconstruções incluem transferência microvascular livre de tecido ósseo ou osteocutâneo de uma variedade de locais, incluindo a fíbula, escápula, e crista ilíaca (DAVID EF, et al., 2016; CHOUINARD AF, et al., 2016).

\section{Prevenção no atendimento odontológico pré e pós radioterapia}

Devido a alterações oriundas causadas pelos efeitos colaterais da RT nos tecidos moles e duros da boca e áreas adjacentes resultado frequente da interação da radiação ionizante com o tecido é de suma importância a atuação do cirurgião - dentista devendo ser necessária em todas as fases da RT. O periodonto do paciente que foi submetido a radioterapia deve ser mantido em boas condições e para isso é necessário a realização rotineira de procedimentos antes e durante a irradiação, pois é a manutenção da saúde oral do paciente oncológico submetido por radioterapia que irá lhe garantir melhor qualidade de vida (SARI J, et al., 2014).

A anamnese e avaliação da condição bucal junto a orientação das técnicas de higiene oral são essenciais para alcançar o melhor prognóstico. Recomenda-se que seja feito toda a adequação de meio bucal prévio ao tratamento radioterápico, como extrações dentárias, restaurações, avaliação da condição periodontal, importância do dente na cavidade, tratamento endodôntico, remoção de restaurações de amálgama e de dentes impactados (ROLIM AEH, et al., 2011). 
A abundância de placa bacteriana amplia a gravidade das infecções das mucosas, podendo levar a um quadro de gengivite. Sendo assim, o controle de infecções na cavidade oral é complementar ao próprio tratamento com a radioterapia, pois infecções não controladas podem comprometer o grau de sucesso da terapia, considerando também que o uso de fármacos antimicrobianos pode causar problemas aos pacientes. Devemos salientar que são indispensáveis a orientação e a forma adequada sobre a higienização oral desses pacientes e saber o protocolo clínico no cuidado odontológico (CARVALHO DA, et al., 2019).

O protocolo clínico no cuidado odontológico da mucosite é: Higiene e prevenção de infecções bucais, laserterapia de baixa intensidade, bochechos com benzidamina em pacientes com doses até 50 Gy na RT, controle da dor com bochechos de morfina a $0,2 \%$ ou doxepina a $0,5 \%$, suplemento oral de zinco porém atenta-se para paciente tabagistas que não deve ser usado; Digeusia: Sulfato zinco $50 \mathrm{mg}$, três vezes ao dia, desde o início até um mês após radioterapia; Xerostomia: Nos pacientes que apresentam algum parênquima salivar funcional, orientação para estimulação salivar gustatória e mastigatória, não existe forte evidência científica para uso de sialogogos no tratamento da hipossalivação após a radioterapia (TORRES SR e GOMES A, 2016).

Contudo, o betanecol pode ser usado de forma preventiva, durante a radioterapia além dos substitutos salivares (saliva artificial); Osteorradionecrose: Higiene oral, próteses bem adaptadas e suspensão do etilismo e tabagismo, irrigação com soluções antimicrobianas nas áreas expostas, antibioticoterapia em infecções agudas, terapia com oxigenação hiperbárica, sequestrectomia e debridamento na região de osso necrótico, a combinação de vitamina E/pentoxifilina/clodronato mostrou-se eficaz na cicatrização da área de exposição óssea, embora estudos controlados sejam necessários para confirmação (TORRES SR e GOMES A, 2016).

\section{Prevenção pré-radioterapia}

A atenção com a higiene bucal prévia ao tratamento da radioterapia deve ser rigorosa, isso incluem visitas regulares ao dentista para exame, profilaxia e aplicação de flúor, além dos cuidados realizados em casa. De acordo com alguns autores, o intervalo de tempo ideal para a adequação do meio bucal é de 21 dias antes da radioterapia (SARI J, et al., 2014; PINTO RFS, 2017; BORGES BS, et al., 2018; MACEDO DR e ANJOS ACY, 2019).

Devemos procurar eliminar toda infecção intraoral realizando tratamento periodontal, restaurações, exodontias e instruções de higiene oral adequada sempre que necessário. Realizar procedimentos invasivos idealmente até três semanas antes de da radioterapia e em casos de exodontias: minimizar o traumar, optar pelo fechamento por primeira intenção. Alveoloplastia, evitando bordas ósseas cortantes. Prevenção da hipossalivação com betanecol (SARI J, et al., 2014; PINTO RFS, 2017; BORGES BS, et al., 2018; MACEDO DR e ANJOS ACY, 2019).

\section{Durante a radioterapia}

No período em que o paciente está em radioterapia os esforços estão voltados para avaliação semanal, foco na manutenção da saúde intraoral e o controle de possíveis alterações da cavidade bucal decorrentes da radioterapia, vale ressaltar que procedimentos invasivos são contraindicados durante a radioterapia (BORGES BS, et al., 2018).

\section{Pós - radioterapia}

Após a conclusão da radioterapia devemos estar atentos para as possíveis alterações e ter uma resposta imediata quanto a prevenção, sendo que é nessa fase que ocorre o aparecimento de possíveis sequelas bucais pós-radioterapia. Devemos evitar procedimentos invasivos para prevenir a osteorradionecrose, se houver necessidade de realização de procedimentos cirúrgicos: realizar profilaxia antibiótica, seguida de antibioticoterapia por sete dias e orientar o paciente sobre risco de osteorradionecrose. A autorização para realização do procedimento pelo paciente, através de assinatura no termo de consentimento é indispensável. Deve-se evitar traumatizar a mucosa oral, eliminando próteses mal adaptadas, dentes com bordas cortantes entre outros, e sempre estar atento na prevenção, controle de infecções e controle da hipossalivação (TORRES SR e GOMES A, 2016; PINTO RFS, 2017). 
Para Lopes LD, et al. (2016) quanto ao protocolo de tratamento, a maioria dos estudos seguem as mesmas normas, procedimentos e conduta de forma que não existe muita variação nos resultados obtidos sobre as terapias. O manejo clínico realizado em casos de complicações orais devido a terapias de tratamento oncológico na região de câncer de cabeça e pescoço consiste primeiramente em cuidados paliativos e prevenção de infecções,, entre elas estão: a prática de higiene oral, os bochechos com os colutórios adequados, lubrificação labial, a nutrição adequada, o controle da xerostomia, a crioterapia, a utilização de laser de baixa potência e a suspensão de substâncias e alimentos irritantes para a mucosa como o tabaco.

De acordo com Curra M, et al. (2018), a mucosite oral é uma resposta aguda ao tratamento que afeta a maioria dos pacientes que recebem radioterapia. Segundo Menezes AC, et al. (2014) o diagnóstico se dá baseado nas manifestações clínicas e é preciso ter conhecimento abrangente para um correto diagnóstico, tendo um diferencial para indicar outras possíveis condições patológicas, o que em alguns casos pode ser complicado pelo fato do sítio ser propício a infecções secundárias de bactérias, vírus e fungos.

Para Lino MDMC, et al. (2011), a mucosite oral é uma resposta inflamatória da mucosa oral a radiação ionizante, utilizadas no tratamento do câncer de cabeça e pescoço, o tratamento inclui cuidados orais básicos, enxágues orais, analgésicos, antibióticos, crioterapia, até o momento não existe um tratamento único capaz de prevenir ou tratar a mucosite de forma eficaz. Recentemente, a fototerapia a laser tem sido utilizada tanto na prevenção quanto no tratamento da mucosite oral, devido à sua capacidade de induzir efeitos biológicos, como analgesia e modulação do processo inflamatório.

Para Sari J, et al. (2014), a xerostomia é uma complicação comum e persistente decorrente da radioterapia em cabeça e pescoço que consiste na diminuição da secreção de saliva. Uma solução flexível para o tratamento da xerostomia seria o uso de estimuladores de glândulas salivares, como a pilocarpina, ou substitutos da saliva ou saliva artificial, que são substâncias paliativas que aliviam o desconforto causado pela xerostomia. O uso frequente de enxaguatório bucal, goles de água e também gomas de mascar sem açúcar ajudam a controlar parcialmente a xerostomia induzida pela radiação.

A osteorradionecrose é caracterizada pela exposição de tecido ósseo necrótico associada a sinais e sintomas como parestesia, infecção secundária e formação de fístula com destruição progressiva do osso e desenvolvimento de fraturas patológicas. $O$ tratamento da osteorradionecrose consiste em intervenção cirúrgica, desbridamento e tratamento da ferida, antibioticoterapia e oxigenação hiperbárica. Se a osteorradionecrose for diagnosticada precocemente, o desbridamento local e o tratamento com antibióticos podem ser bem-sucedidos (DAVID EF, et al., 2016).

\section{CONSIDERAÇÕES FINAIS}

Pacientes que serão submetidos a radioterapia de cabeça e pescoço necessitam de acompanhamento do cirurgião-dentista antes, durante e após o tratamento, tendo em vista que alterações significativas podem ocorrer na região oral devido ao tratamento antineoplásico. Portanto torna-se fundamental o acompanhamento odontológico, sendo de suma importância que anteceda o tratamento radioterápico como forma de prevenir e diminuir a incidência de complicações bucais e infecções oportunistas.

\section{REFERÊNCIAS}

1. BARBIERI T, et al. Alternativas atuais na prevenção e tratamento da xerostomia decorrente dos tratamentos antineoplásicos. Revista Gaúcha de Odontologia, 2020; 68(8).

2. BARROS O, et al. Disgeusia: a propósito de um caso clínico. Revista Portuguesa de Medicina Geral e Familiar, 2015; 31(6): 272-276.

3. BORGES BS, et al. Atendimento odontológico de paciente submetido à radioterapia em região de cabeça e pescoço: relato de caso clínico. Revista Odontológica Universidade da Cidade de São Paulo, 2018; 30(3): 332-340.

4. CARVALHO DA, et al.Prevenção e manejo terapêutico da osteoradionecrose dos maxilares: revisão de literatura. Revista Odontológica de Araçatuba, 2019; 40(3): 38-44.

5. CHOUINARD AF, et al. Hyperbaric Oxygen Therapy for Head and Neck Irradiated Patients with Special Attention to Oral and Maxillofacial Treatments. J Can Dent Assoc, 2016; 82(24): 1-5.

6. CURRA M, et al. Protocolos quimioterápicos e incidência de mucosite bucal. Journal Einstein, 2018. 6(1). 
7. DAVID EF, et al. Manejo terapêutico e preventivo da osteorradionecrose: revisão integrativa da literatura. Revista brasileira de odontologia, 2016; 73(2): 150-156.

8. DIAS LL, et al. Osteorradionecrose dos maxilares: um estudo descritivo. Anais Seminário de Iniciação Científica SEMIC, 2018; 0(22): 1-4.

9. EDUARDO FP, et al. Influência dos cuidados odontológicos acompanhados de laserterapia sobre a mucosite oral durante transplante alogênico de células hematopoiéticas: estudo retrospectivo. Journal Einstein, 2011. 9(2): 201206.

10. FRANCO R, MARTA GNT. Timing factors as prognostic variables in patients with head and neck squamous cell carcinoma treated with adjuvant radiotherapy: a literature review. Revista Associação Médica Brasileira, 2020; 66(3): 380-384.

11. FREITAS DA, et al. Sequelas bucais da radioterapia de cabeça e pescoço. Revista CEFAC, 2011; 13(6): $161-171$.

12. HOLMES TSV, et al. Fatores relacionados ao surgimento e gradação da mucosite oral radioinduzida. Revista Cubana de Estomatología, 2014; 51(1): 71-79.

13. LINO MDMC, et al. Fototerapia a laser como tratamento para mucosite oral induzida por radioterapia. Braz. Dent. J, 2011; 22(2): 162-165.

14. LOPES LD, et al. Prevenção e tratamento da mucosite em ambulatório de oncologia: uma construção coletiva. Texto contexto -enfermagem, 2016; 25(1): 1-9.

15. MACEDO DR, ANJOS ACY. Experiência de radioterapia na cabeça e pescoço. Revista Gaúcha Odontológica, 2019. 67.

16. MENEZES AC, ROSMANINHO AE. Abordagem clínica e terapêutica da mucosite oral induzida por radioterapia e quimioterapia em pacientes com câncer. Revista brasileira odontológica, 2014; 71(1): 35-8.

17. NEVILLE B, et al. Patologia Oral e Maxilofacial. 4 ed. Rio de Janeiro: Editora Elsevier, 2016; $296 \mathrm{p}$.

18. PINTO RFS. Abordagens terapêuticas na osteorradionecrose dos maxilares. Dissertação (Mestrado em Medicina Dentaria) - Faculdade de Medicina Dentária da Universidade do Porto, Portugal, 2017; 46p.

19. REOLON LZ, et al. Impacto da laserterapia na qualidade de vida de pacientes oncológicos portadores de mucosite oral. Revista Odontológica UNESP, 2017; 46(1): 19-27.

20. RIBEIRO GH, et al. Osteonecrosis of the jaws: a review and update in etiology and treatment. Braz J Otorhinolaryngol, 2018; 84(1):102-108.

21. ROLIM AEH, et al. Repercussões da radioterapia na região orofacial e seu tratamento. Radiologia Brasileira, 2011; 44(6): 388-395.

22. SARI J, et al. Oral complications in patients receiving head and neck radiation therapy: a literature review. Revista Gaúcha de Odontologia, 2014; 62(4): 395-400.

23. TORRES SR, GOMES A. Cuidados Odontológicos ao Paciente Submetido à Radioterapia (Parte II). Revista Eletrônica CRO-RJ, 2016; 28(3): 1. 